\title{
Navegación Basada en Prestaciones: Aprendizaje Basado en Proyectos para estudiantes de Aeronavegación.
}

\begin{abstract}
V.P. Cuenca-Gotor ${ }^{a}$ P. Yuste Pérez ${ }^{b}$, J.A. Vila Carbóc ${ }^{c}$, I. Despujol Zabalad ${ }^{d}$ J.A. Monsoriu-Serra ${ }^{\mathrm{e}}$ ${ }^{a}$ Departamento de Física Aplicada, Escuela Técnica Superior de Ingeniería del Diseño (ETSID), Universitat Poitècnica de València (UPV), vacuego@fis.upv.es; ${ }^{b}$ Departamento de Informática de Sistemas y Computadores, ETSID, UPV, pyuste@disca.upv.es; 'Departamento de Informática de Sistemas y Computadores, ETSID, UPV, jvila@disca.upv.es; dDepartamento de Ingeniería de la Construcción y de Proyectos de Ingeniería Civil, ETSID, UPV, ndespujol@asic.upv.es; y ${ }^{\mathrm{D}}$ Departamento de Física Aplicada, ETSID, UPV, jmonsori@fis.upv.es.
\end{abstract}

\section{\$EWWDWW}

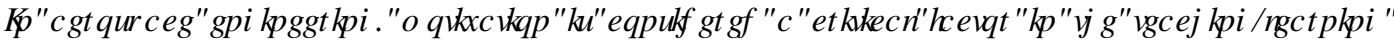

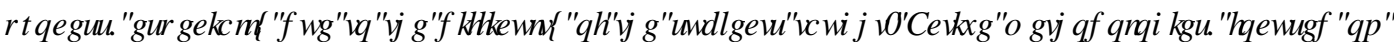

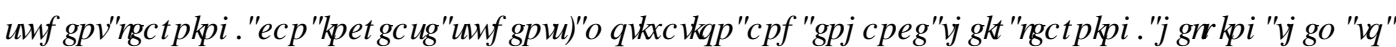

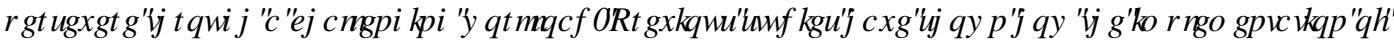

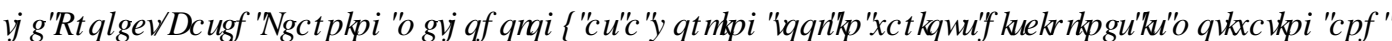

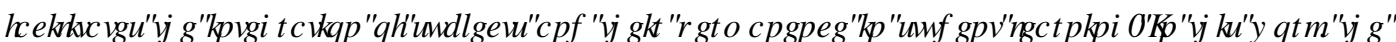

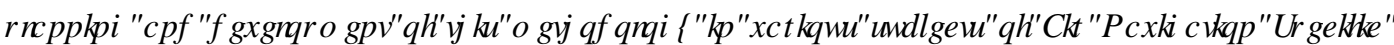
7 HFKQR

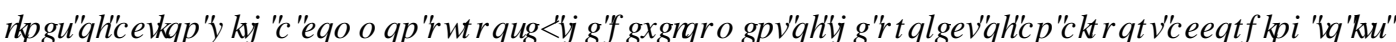

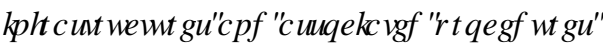

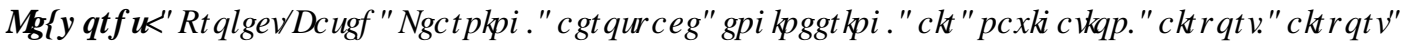

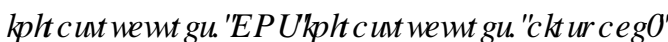

[1]

\section{HXP HQ}

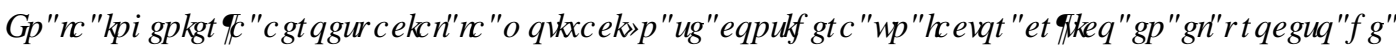

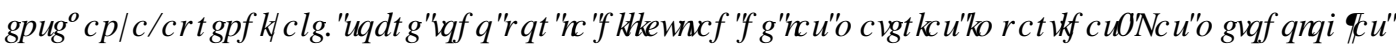

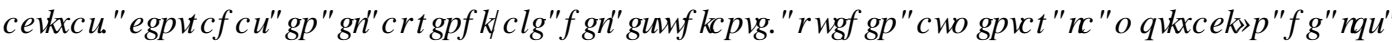

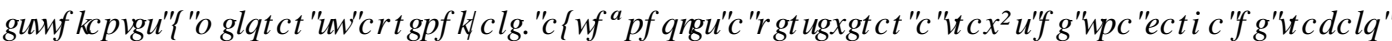

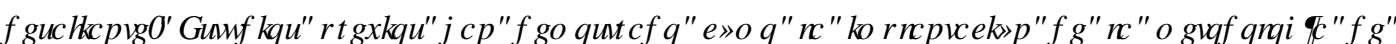

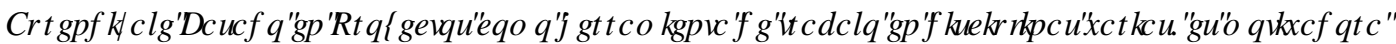

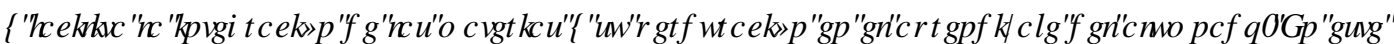

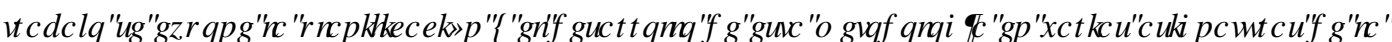

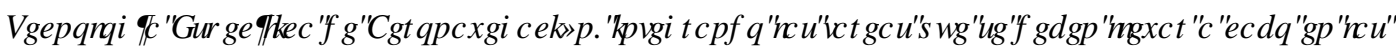

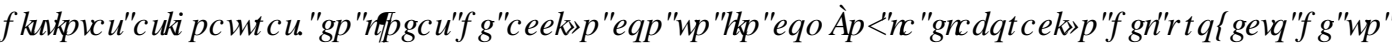

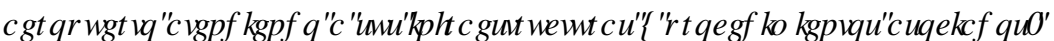

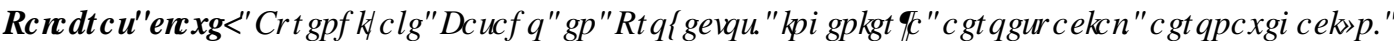

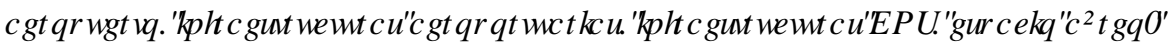




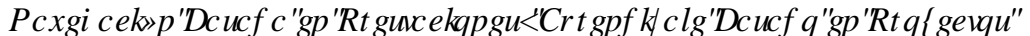 SDWHMKGDQUAVGHS HRQDYHDFI QD}

\section{Introducción}

El Grado en Ingeniería Aeroespacial (UPV, 2021a) culmina la formación científico-técnica de su alumnado con el estudio de intensificación de una de sus tecnologías específicas (Aeromotores, Aeronaves o Aeronavegación), que habilita al estudiante para el ejercicio de la profesión de la ingeniería técnica aeronáutica, y que se desarrolla entre el sexto y el octavo semestre del plan de estudios. Las asignaturas ofertadas en el módulo 8 - Tecnología Específica de Aeronavegación (UPV, 2021b) entroncan con asignaturas de carácter obligatorio y con otras afines de carácter optativo, ofreciendo a los estudiantes una formación completa que les permitirá desarrollar su labor profesional en el ámbito general de la ingeniería técnica aeronáutica y, en particular, de esta intensificación. Las asignaturas implicadas en el desarrollo de este trabajo son las siguientes (7DEDD ):

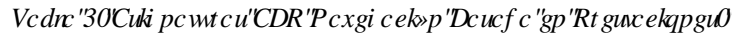

\begin{tabular}{llcccc}
\hline Nombre - código & Tipo & Curso & Sem. & ECTS & N est. $^{\mathbf{2}}$ \\
\hline $\begin{array}{l}\text { Planificación y Desarrollo de } \\
\text { Aeropuertos - 11922 }\end{array}$ & Optativa & 3 & 5 & 6 & 14 \\
$\begin{array}{l}\text { Navegación Aérea, Cartografía y } \\
\text { Cosmografía - 11935 }\end{array}$ & Optativa & 3 & 6 & 4,5 & 26 \\
$\begin{array}{l}\text { Infraestructuras para la Navegación } \\
\text { Aérea - 11943 }\end{array}$ & Optativa & 4 & 7 & 6 & 22 \\
$\begin{array}{l}\text { Gestión del Espacio Aéreo I - 11936 } \\
\text { Ingeniería de los Sistemas de }\end{array}$ & Optativa & 4 & 7 & 6 & 26 \\
Navegación Aérea II - 11940 & Optativa & 4 & 8 & 6 & 27 \\
\hline \begin{tabular}{l} 
Gestión del Espacio Aéreo II - 11937 \\
\hline
\end{tabular} & Optativa & 4 & 8 & 6 & 31 \\
\hline
\end{tabular}

En la situación de partida, la organización de los estudios presentados conllevaba la realización de múltiples tareas en las distintas asignaturas, que facilitaban la adquisición de los conocimientos y competencias necesarios para el devenir profesional del alumnado, y que formaban parte de la evaluación final de las asignaturas, junto con la realización de pruebas escritas de respuesta abierta. La diversificación, y en ocasiones duplicidad, de estas tareas requería una dedicación extra del alumnado, que se enfrentaba a estas tareas con poca motivación.

En el caso de la formación en ingeniería aeroespacial, se considera la motivación como factor crítico en el proceso de aprendizaje y en el rendimiento académico del alumnado, sobre todo debido a la dificultad de las materias impartidas (López, 2019). Es importante, por tanto, identificar las metodologías activas que pueden aumentar la motivación de los estudiantes y mejorar su aprendizaje, ayudándoles a perseverar a través de una carga de trabajo desafiante (Prince, 2004).

Las metodologías activas de aprendizaje se basan en actividades que implican a los estudiantes de tal manera que realizan las tareas con el pensamiento aplicado en la tarea desarrollada, obteniendo una conjunción entre actividad y reflexión que mejora la capacidad de aprendizaje del alumnado. Frente al aprendizaje tradicional, en que el profesor lidera el trabajo de los estudiantes mediante la impartición de clases magistrales de teoría y aplicaciones prácticas dirigidas, cada vez más se demanda el papel 
protagonista del alumnado en el proceso de enseñanza-aprendizaje, sin que por ello haya que perder de vista la importancia de la lección magistral en el principio de todo proceso, junto con el trabajo en equipo y el trabajo autónomo del estudiante (Zabalza, 2011). Se hace necesario, pues, seguir el camino hacia un modelo docente más efectivo centrado en el aprendizaje, sobre todo en el aprendizaje autónomo del estudiante tutorizado por el profesorado, y centrado en los resultados de aprendizaje; un modelo que enfoque el proceso de enseñanza-aprendizaje como un trabajo cooperativo entre profesorado y alumnado (Fernández, 2006).

La aplicación de nuevos modelos centrados en la práctica beneficia particularmente a la educación en ingeniería por su componente de práctica esencial. Además, las asignaturas de segundo ciclo, y en concreto las de tecnologías específicas, que tienen un carácter muy tecnológico y sistemático, son más adecuadas para implementar métodos de aprendizaje activo como el Aprendizaje Basado en Proyectos (ABP), que será la herramienta clave para este proceso (Rodríguez, 2015). El ABP potencia no solo la adquisición por parte de los estudiantes de las competencias específicas de cada asignatura, sino también el desarrollo de competencias o habilidades como la capacidad para resolver problemas, el pensamiento crítico, la adaptabilidad, la capacidad para la recogida de datos, el trabajo en equipo, la proactividad, etc., cada vez más valoradas en el ámbito profesional de la aviación y la aeronáutica (McGunagle, 2018).

De hecho, de entre los beneficios de este método se destaca que: aumenta el aprendizaje autónomo, prepara a los estudiantes para los puestos de trabajo, aumenta la motivación, fortalece la autoconfianza, establece la conexión entre el aprendizaje en la escuela y la realidad, ofrece oportunidades de colaboración para construir conocimiento, aumenta las habilidades sociales y de comunicación, aumenta las habilidades para la solución de problemas... Si a esto añadimos los beneficios del trabajo colaborativo en cuanto a aumento de la interacción y de la habilidad de pensamiento crítico en el negociado de soluciones, el ABP parece ser una buena estrategia para la mejora de la calidad del aprendizaje (Tippelt, 2001; Maldonado, 2008).

La unificación de las tareas que se deben llevar a cabo en las distintas asignaturas implicadas en el desarrollo de este trabajo, en líneas de acción con un fin común, permitirá al estudiante ser el que lidere y dirija su propio proyecto, requiriendo la toma de decisiones propia aun contando siempre con el apoyo del profesorado. Será este último quien inicie el proceso identificando los requisitos y las competencias a adquirir mediante los contenidos y habilidades definidas, siendo el estudiante autónomo en el aprendizaje de los contenidos y desarrollo de las habilidades. Además, se fomentará también el trabajo de proyectos por equipos utilizando las listas de requisitos como interfaz entre equipos.

\section{Objetivos}

El fin último de este proyecto es mejorar el proceso de enseñanza-aprendizaje de los estudiantes de la Tecnología Específica de Aeronavegación (Grado en Ingeniería Aeroespacial), empleando la metodología de ABP. Para ello, los estudiantes elaborarán el diseño de un aeropuerto atendiendo a sus infraestructuras y procedimientos asociados.

Los objetivos específicos, a lograr por parte del profesorado, son:

- Diseñar actividades de aprendizaje abiertas, basadas en casos reales, asociadas a la entrega del proyecto y coordinadas entre asignaturas, empleando el proyecto como nexo de unión. Estas actividades facilitarán y mejorarán el proceso de aprendizaje y evaluación de las competencias generales, específicas y transversales.

- Planificar el desarrollo de estas actividades, en cuanto al establecimiento de los tiempos de trabajo y fechas de entrega.

(cc) EY-NC-ND 2021, Universitat Politècnica de València

CRQJUHRR, Q15 HGस००स० 


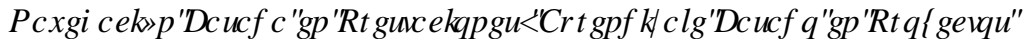 SDWHMKGDQUARCHS HRQDYHDFIY}

[

- Realizar el seguimiento del trabajo del alumnado, analizando y valorando los resultados obtenidos, tanto respecto a la satisfacción con el método de ABP como herramienta de trabajo, como al logro de los resultados de aprendizaje.

- Visualizar los resultados de las actividades realizadas por el alumnado mediante la organización de jornadas de exposición. En la presentación de los proyectos desarrollados por los estudiantes se fomentará la participación activa de los mismos y el intercambio y aporte de ideas.

Respecto a los estudiantes, se espera que, a la consecución del proyecto, sean capaces de:

- Recabar la información necesaria en bibliografía y normativa aplicable al diseño de aeropuertos y cálculo de infraestructuras.

- Identificar los elementos funcionales básicos del sistema de navegación aérea y las necesidades del equipamiento embarcado y terrestre para una correcta operación.

- Aplicar los conocimientos teóricos de las materias implicadas a la práctica profesional, mediante el diseño del lado tierra y el lado aire de un aeropuerto.

- Calcular los elementos necesarios para la definición de un aeropuerto: parámetros de las pistas aéreas, redes de infraestructuras eléctricas y sistemas específicos de la aeronavegación.

- Elaborar el informe del diseño de un aeropuerto, incluyendo toda la documentación necesaria, para su presentación en formato de comunicación oral (ponencia o póster).

\section{Desarrollo de la innovación}

Para lograr el objetivo principal del presente proyecto, el estudiante completará el diseño de un proyecto de infraestructuras y procedimientos asociados a un aeropuerto desde sus diferentes puntos de vista: diseño de las infraestructuras aeroportuarias y de las redes de comunicaciones, navegación y vigilancia (CNS), y diseño del espacio aéreo; intentando que la visión del alumnado sea integradora y no por componentes aislados. En el desarrollo del proyecto se han programado actividades a llevar a cabo individualmente y en equipos de entre 4 y 5 estudiantes.

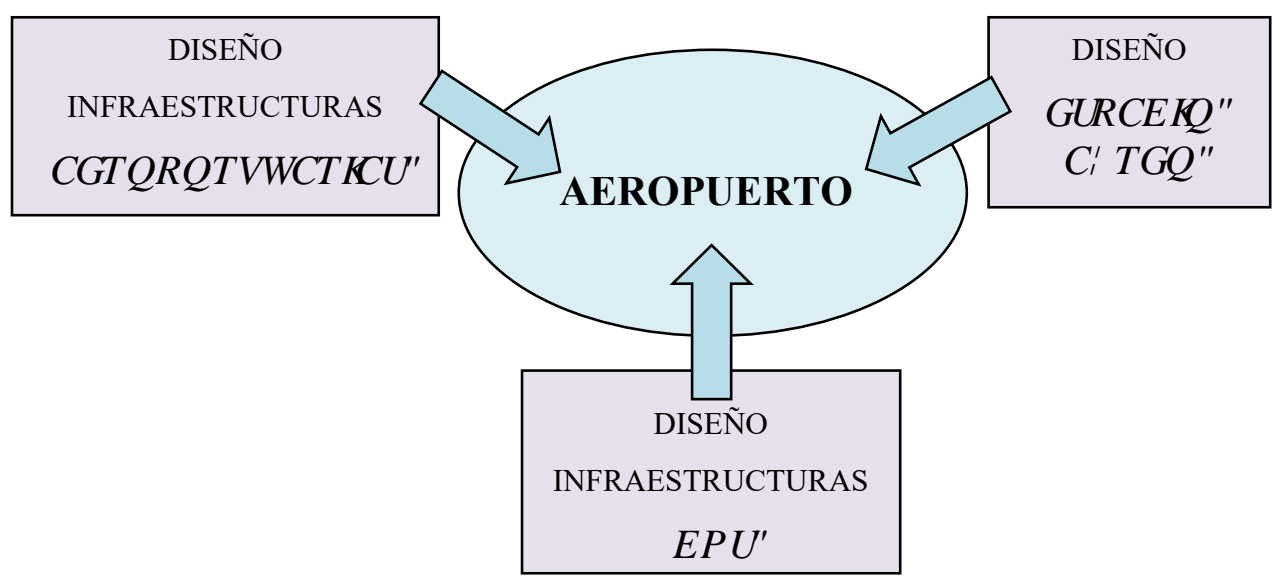

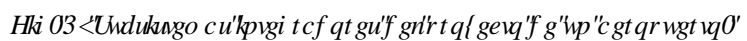

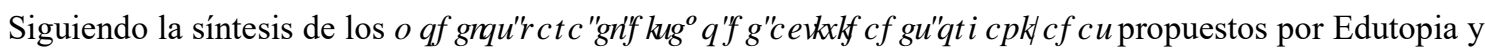
Buck Institute for Education (García, 2017), en primer lugar se abordó el "Desarrollo de la idea del Proyecto" (FASE 1), a continuación se llevó a cabo la "Preparación del Soporte" y la "Planificación de Actividades" (FASE 2), y se finalizará con la "Presentación Final del Proyecto y Evaluación" (FASE 3). 
En este momento no se cuenta con la evaluación final del proyecto, pues los estudiantes se encuentran aún en fase de realización de varias de las actividades; sin embargo, sí que se cuenta ya con los instrumentos de evaluación de los resultados, tanto en cuanto a la evaluación del proyecto final como respecto al grado de satisfacción del alumnado en el desarrollo del mismo.

\subsection{FASE 1: Desarrollo de la idea del Proyecto.}

Como se ha indicado en el apartado anterior, el objetivo principal de este proyecto es mejorar el proceso de enseñanza-aprendizaje de los estudiantes empleando la metodología de ABP. Para ello, se ha partido de un planteamiento abierto y significativo para los estudiantes de esta tecnología específica, como es la elaboración del diseño de un aeropuerto atendiendo a sus infraestructuras y procedimientos asociados.

Ante la autenticidad de este reto, y su alto nivel en la escala de niveles de conocimiento, se pretende que los estudiantes sean capaces de demostrar sus conocimientos en todas las materias necesarias para la consecución del proyecto. Además, al ser un proyecto complejo, se debe desarrollar mediante diferentes actividades y la síntesis de la información recabada en todas ellas.

\subsection{FASE 2: Preparación del Soporte y Planificación de Actividades.}

El profesorado participante ha diseñado el proyecto y elaborado el material necesario para su puesta en marcha, seguimiento y evaluación. Dado que existe una secuenciación temporal de las asignaturas implicadas en el proyecto prevista en el propio Plan de Estudios (7DEDD), el desarrollo del trabajo se está ajustando al propio desarrollo de la adquisición de competencias por parte del alumnado. La implicación de las asignaturas en cada uno de los subsistemas mostrados en el diagrama anterior () $U \amalg$ ), así como los resultados esperados de cada una de las actividades llevadas a cabo por parte del alumnado, se muestran a continuación:

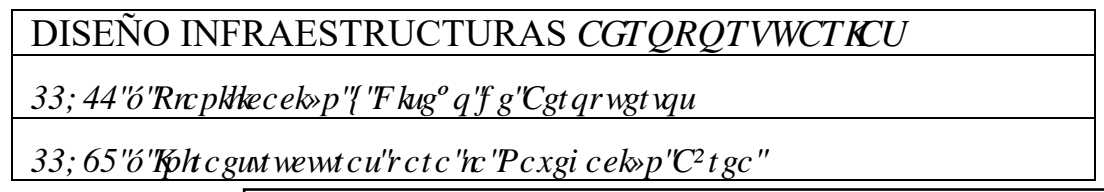

RESULTADOS:

- Establecimiento y análisis de requisitos del subsistema Diseño de Infraestructuras Aeroportuarias.

- Proyecto de pistas.

- Proyecto de balizamiento.

- Proyecto de instalaciones eléctricas.

\begin{tabular}{|c|}
\hline DISEÑO INFRAESTRUCTURAS \&1 6 \\
\hline 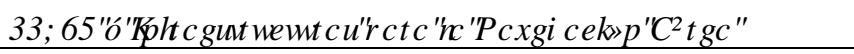 \\
\hline 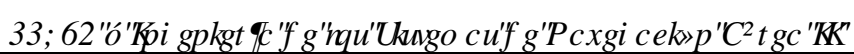 \\
\hline
\end{tabular}

RESULTADOS:

- Establecimiento y análisis de requisitos del subsistema Diseño de Infraestructuras CNS.

- Proyecto de radioayudas.

- Proyecto de instalaciones radar.

- Proyecto de comunicaciones. 


\begin{tabular}{|c|}
\hline DISEÑO ( 63\$ \&,2 a\$e 5 ( 2 \\
\hline 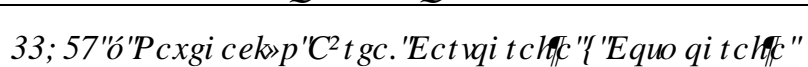 \\
\hline
\end{tabular}

RESULTADOS:

- Establecimiento y análisis de requisitos del subsistema Diseño del Espacio Aéreo.

- Estudio de recepción de señales radioeléctricas de satélites.

- Cartas de navegación con el diseño de los procedimientos de aproximación al aeropuerto.

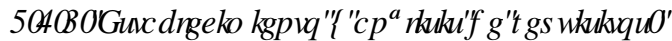

Estos subsistemas no son independientes entre sí; por ejemplo, el Diseño del Espacio Aéreo depende de las Infraestructuras CNS existentes o, a la inversa, si se pretende realizar un determinado Diseño de Espacio Aéreo deberán generarse una serie de requisitos para las infraestructuras que deberán soportarlo. En la metodología propuesta el estudiante debe aprender que, para realizar un diseño, deben establecerse unos requisitos sobre cada subsistema y que el punto de partida para el diseño es un análisis detallado de estos requisitos. Asimismo, el diseño de un subsistema siempre requiere establecer una lista de requisitos sobre los subsistemas en los cuales se sustenta. El establecimiento y análisis de requisitos sobre cada uno de los subsistemas resulta así una actividad clave en la metodología propuesta.

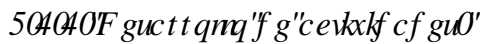

En la asignatura 11922 - Planificación y Diseño de Aeropuertos, que se imparte en quinto semestre, los estudiantes, trabajando en modo grupal, preparan un informe estratégico sobre la posible instalación de un aeropuerto para la ciudad de Cáceres, con el apoyo del Manual de Diseño de Aeródromos (OACI, 2006a), empleando el Boeing 737-800 como avión de diseño. En este informe se evalúa el tamaño de pista necesario en función del avión de diseño y la estimación del resto de las necesidades del terreno, y se ha realizado la selección de la zona en la que instalar el aeropuerto teniendo en cuenta el espacio disponible para la construcción del aeropuerto, la situación de los obstáculos, las variables medioambientales y geográficas de la zona, la situación de las poblaciones, la situación de las vías terrestres, etc. A pesar de que la asignatura optativa es común a estudiantes de las diferentes tecnologías específicas, se realiza un seguimiento particular de los trabajos del alumnado que curse la tecnología específica de Aeronavegación.

Posteriormente, en la asignatura 11935 - Navegación Aérea, Cartografía y Cosmografía, optativa propia de la tecnología específica de Aeronavegación impartida en sexto semestre, los equipos de estudiantes trabajan en la validación de procedimientos de navegación basados en satélite (SBAS). Para ello se requiere conocer el espacio físico en el que se hará la validación y, a partir de él, estudiar la recepción de las señales radioeléctricas procedentes de los satélites en esa zona (Pisacane, 2008). Dado que se cursa en sexto semestre, se secuencian las fases del trabajo de manera que el estudiante realiza el estudio de validación de la zona en la que se ha implantado el aeropuerto.

En la asignatura 11943 - Infraestructuras para la Navegación Aérea, que se imparte en séptimo semestre, se trabajan todas las instalaciones del aeropuerto propuesto en la asignatura 11922 - Planificación y Diseño de Aeropuertos, tanto las necesarias para el funcionamiento del aeropuerto (central eléctrica, red de distribución y canalizaciones eléctricas) como las utilizadas por los sistemas de ayuda a la navegación (balizamiento, ILS, NDB, VOR Doppler, DME...). Para ello se emplean los documentos de la Organización 
de Aviación Civil Internacional (OACI) referentes a sistemas eléctricos y ayudas visuales en el diseño de aeródromos (OACI, 1983; OACI, 2004; OACI, 2009). Como continuación de la parte del proyecto desarrollada en esta asignatura, en el siguiente semestre, la asignatura 11940 - Ingeniería de los Sistemas de Navegación Aérea II trabajará todas las infraestructuras de navegación no basadas en tierra, es decir, de navegación basada en satélite (GNSS). En esta asignatura se aprovechará el estudio de validación realizado en la asignatura 11935 - Navegación Aérea, Cartografía y Cosmografía para completarlo mediante el estudio desde el punto de vista del satélite (estructura de la señal, mensaje de navegación, posicionamiento y sistemas de aumentación basados en GNSS), todo ello basado en la tecnología de los sistemas de comunicación vía satélite (Maral, 2005).

Las dos últimas asignaturas implicadas en el proyecto abordan un enfoque de las infraestructuras y procedimientos asociados al aeropuerto diseñado desde el punto de vista de los servicios para la navegación aérea. En primer lugar, la asignatura 11936 - Gestión del Espacio Aéreo I, de séptimo semestre, se centra en el servicio de vigilancia: en función de los requisitos del aeropuerto definido en la asignatura 11922 Planificación y Diseño de Aeropuertos, se estudia la implantación de un servicio de vigilancia basado en radar primario, secundario y/o ADSB (OACI, 2011; Sáez, 2012). Por último, en la asignatura 11937 Gestión del Espacio Aéreo II, de octavo semestre, los estudiantes elaborarán un informe con el diseño de los procedimientos de vuelo para el aeropuerto, en base a la disponibilidad de las infraestructuras de navegación aérea diseñadas en las asignaturas anteriores y utilizando los criterios de prestaciones de navegación y franqueamiento de obstáculos establecidos por la OACI (OACI, 2006b).

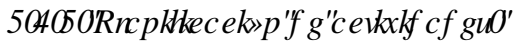

La preparación del proyecto por parte del profesorado ha conllevado una serie de tareas previas como la planificación temporal de las actividades a llevar a cabo por el alumnado, las entregas y exposiciones previstas; el diseño del sistema de evaluación final del proyecto y la elaboración de los sistemas de recogida de resultados. Sin embargo, el proyecto se ha tenido que ir adaptando a las circunstancias derivadas de la situación de crisis sanitaria en la que aún estamos inmersos, por lo que parte de los hitos del desarrollo han sido modificados y orientados a la nueva -y casi principal- forma de relación y comunicación.

En la implementación del proyecto se están desarrollando las actividades que se han definido en el apartado

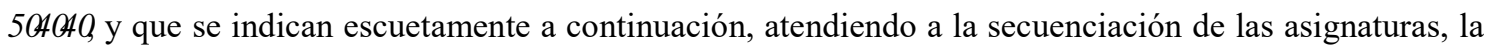
relación que existe entre los subsistemas integradores del proyecto, las actividades que se desarrollan dentro de la asignatura (clases en aula, laboratorio, tutorías) y las distintas metodologías que se usan a lo largo del curso (clases magistrales, resolución de problemas, etc.) (García, 2017):

1. Establecimiento y análisis de requisitos del subsistema Diseño de Infraestructuras Aeroportuarias ( $5^{\circ}$ semestre).

2. Informe de instalación de aeropuerto y proyecto de pistas $\left(5^{\circ}\right.$ semestre $)-\mathrm{A} 1$.

3. Evaluación parcial por parte de los profesores de las asignaturas implicadas ( $5^{\circ}$ semestre).

4. Establecimiento y análisis de requisitos del subsistema Diseño del Espacio Aéreo ( $6^{\circ}$ semestre).

5. Estudio de recepción de señales radioeléctricas de satélites $\left(6^{\circ}\right.$ semestre $)-\mathrm{A} 2$.

6. Evaluación parcial por parte de los profesores de las asignaturas implicadas ( $6^{\circ}$ semestre).

7. Establecimiento y análisis de requisitos del subsistema Diseño de Infraestructuras CNS ( $7^{\circ}$ semestre).

8. Proyecto de balizamiento $\left(7^{\circ}\right.$ semestre $)-\mathrm{A} 3$.

9. Proyecto de instalaciones eléctricas $\left(7^{\circ}\right.$ semestre $)-\mathrm{A} 4$.

10. Proyecto de radioayudas $\left(7^{\circ}\right.$ semestre $)$ - A5.

11. Proyecto de instalaciones radar $\left(7^{\circ}\right.$ semestre $)-\mathrm{A} 6$.

12. Evaluación parcial por parte de los profesores de las asignaturas implicadas ( $7^{\circ}$ semestre).

(cc) EY-NC-ND 2021, Universitat Politècnica de València

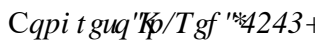




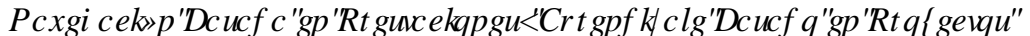 SDWHMKGDQWAVGHS HRQDYH DFIY QD}

\section{[}

13. Proyecto de comunicaciones $\left(8^{\circ}\right.$ semestre $)-A 7$.

14. Cartas de navegación con el diseño de los procedimientos de aproximación al aeropuerto $\left(8^{\circ}\right.$ semestre) - A8.

15. Evaluación parcial por parte de los profesores de las asignaturas implicadas ( $8^{\circ}$ semestre).

16. Exposición y evaluación final del proyecto del aeropuerto $\left(8^{\circ}\right.$ semestre $)$.

\subsection{FASE 3: Presentación Final del Proyecto y Evaluación.}

La conclusión del proyecto de diseño de un aeropuerto se realizará mediante la entrega de un documento que recoja y sintetice los resultados de las actividades desarrolladas a lo largo de los dos cursos. Este documento consistirá en una presentación de diapositivas o un póster para su exposición. En el caso de que se permita hacer una exposición presencial, se optará por la presentación en formato póster, situando los paneles expositores en el patio de la Escuela para que puedan ser visualizados y explicados como si de la jornada de un congreso se tratara. En caso contrario, se optará por la exposición de diapositivas a través de Teams, como ya se ha realizado en actividades anteriores. Los proyectos concluidos podrán considerarse como base para la elaboración de Trabajos Fin de Grado, y la evaluación de estos se computará con un 10\% de la nota de proyecto de las dos últimas asignaturas: Ingeniería de los Sistemas de Navegación Aérea II 11940 y Gestión del Espacio Aéreo II - 11937.

La evaluación de los resultados obtenidos se basa en el seguimiento del proceso enseñanza-aprendizaje del alumnado, mediante la evaluación de las competencias generales y específicas, así como de las competencias transversales trabajadas en el desarrollo del proyecto. A continuación se muestran las actividades que conforman el proyecto, indicándose la metodología empleada para su consecución, las competencias transversales que trabaja, los sistemas seguidos para su evaluación (ICE-UPV, 2021) y el peso de esta en la asignatura, teniendo en cuenta que las actividades A3, A4 y A5 se realizan en la misma asignatura (7DECD):

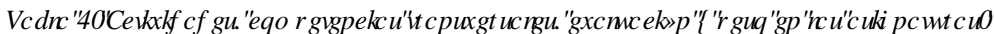

\begin{tabular}{|c|c|c|c|c|}
\hline Activ. & Metodología & CT & Evaluación & Peso \\
\hline A1 & $\begin{array}{l}\text { Trabajo en equipo de instalación de aeropuerto, } \\
\text { con exposición por Teams. }\end{array}$ & $06-08$ & $\begin{array}{l}\text { Calidad del proyecto. } \\
\text { Rúbricas ICE CT06 y CT08. }\end{array}$ & $30 \%$ \\
\hline A2 & $\begin{array}{l}\text { Trabajo en equipo de recepción de señales, con } \\
\text { exposición por Teams. }\end{array}$ & $08-11$ & $\begin{array}{l}\text { Calidad del trabajo. } \\
\text { Valoración de la exposición. }\end{array}$ & $40 \%$ \\
\hline A3 & Trabajo individual de proyecto de balizamiento. & $05-07$ & $\begin{array}{l}\text { Calidad del proyecto. } \\
\text { Rúbrica ICE CT07. }\end{array}$ & $10 \%$ \\
\hline A4 & Trabajo individual de proyecto de red eléctrica. & $05-07$ & $\begin{array}{l}\text { Calidad del proyecto. } \\
\text { Rúbrica ICE CT07. }\end{array}$ & $10 \%$ \\
\hline A5 & Trabajo individual de proyecto de radioayudas. & $05-07$ & $\begin{array}{l}\text { Calidad del proyecto. } \\
\text { Rúbrica ICE CT07. }\end{array}$ & $10 \%$ \\
\hline A6 & Trabajo individual de proyecto de radar. & $09-10$ & Calidad del trabajo. & $20 \%$ \\
\hline A7 & $\begin{array}{l}\text { Trabajo individual de proyecto de } \\
\text { comunicaciones. }\end{array}$ & $01-03$ & $\begin{array}{l}\text { Calidad del trabajo. } \\
\text { Corrección de cálculos. }\end{array}$ & $40 \%$ \\
\hline A 8 & $\begin{array}{l}\text { Trabajo individual con entrega de informe de } \\
\text { procedimientos. }\end{array}$ & $02-13$ & Calidad del informe. & $50 \%$ \\
\hline
\end{tabular}




\section{Resultados}

El nivel de logro de los objetivos del propio proyecto: diseño de las actividades y planificación de las mismas; y análisis del seguimiento del proceso de enseñanza-aprendizaje del alumnado, respecto a los resultados de aprendizaje y respecto al grado de satisfacción con la metodología empleada; no puede ser analizado en su totalidad, al encontrarnos todavía inmersos en él. Los resultados mostrados a continuación hacen referencia a la planificación y desarrollo de la metodología, mientras que el análisis de los resultados de aprendizaje y del grado de satisfacción del alumnado se llevará a cabo a la finalización del presente curso académico 2020/2021.

Para la puesta en marcha y funcionamiento del proyecto se han llevado a cabo las reuniones previstas, realizando siempre un acta de la reunión que permitiera llevar a cabo el seguimiento del diseño e implantación del mismo (,PDJHQ $)$. De esta manera se fue concretando la planificación temporal de las distintas actividades y se facilitó la adaptación de estas a la situación de distanciamiento social requerida por la pandemia. A pesar de las dificultades en el establecimiento de reuniones con la periodicidad deseada, derivadas de la alta carga en gestión e investigación de algunos de los participantes, la coordinación entre el profesorado de las distintas asignaturas ha sido exitosa.

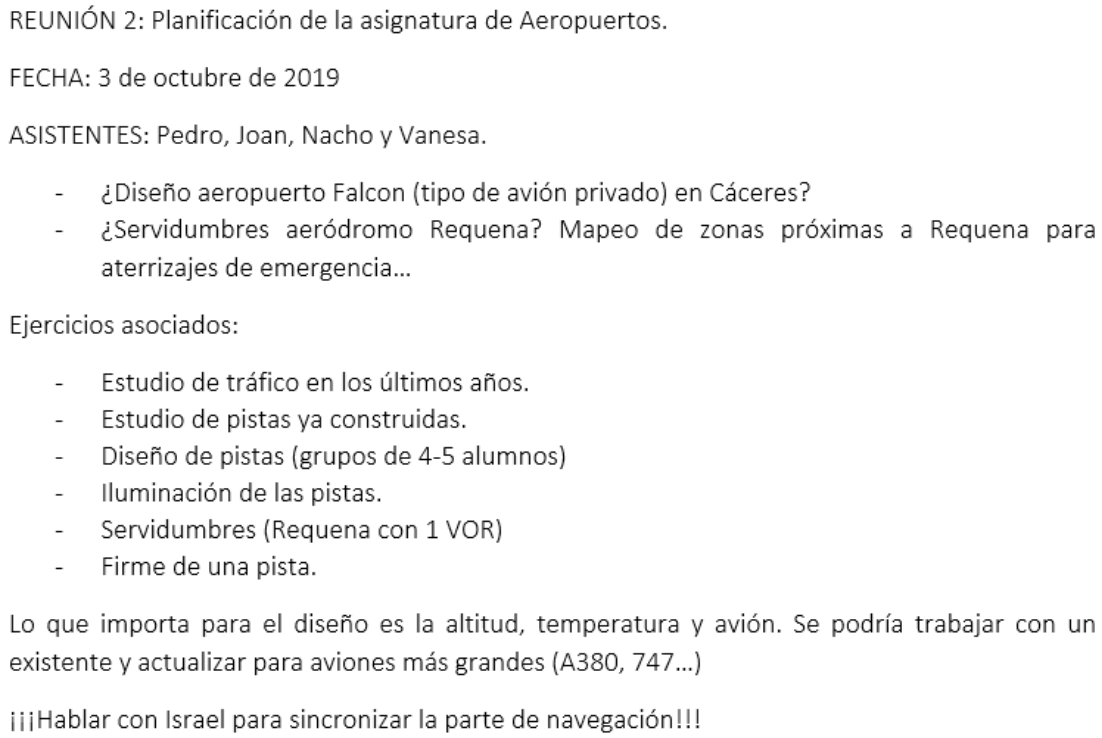

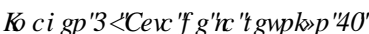

Respecto al análisis del logro de los resultados del proyecto, enfocados en el alumnado, se ha elaborado una rúbrica para la evaluación de la presentación final del proyecto (,PDJWQ). También se ha elaborado una encuesta $(, P D J H Q)$ para la recolección de datos globales con el fin de detectar tendencias en su análisis, con una pregunta de respuesta abierta en la que plasmar las impresiones, problemas, sugerencias de mejora, etc. Mediante esta técnica se analizarán resultados en cuanto a satisfacción del alumnado, tiempo de dedicación, problemas en la consecución del proyecto, etc. para completar la valoración del proyecto (7DE(D) ).

Por último, se considera fundamental la visualización de los resultados, tanto de los proyectos realizados por los estudiantes mediante la organización de jornadas de exposición en cuanto sea posible, como de los propios de la aplicación de la metodología de ABP. 


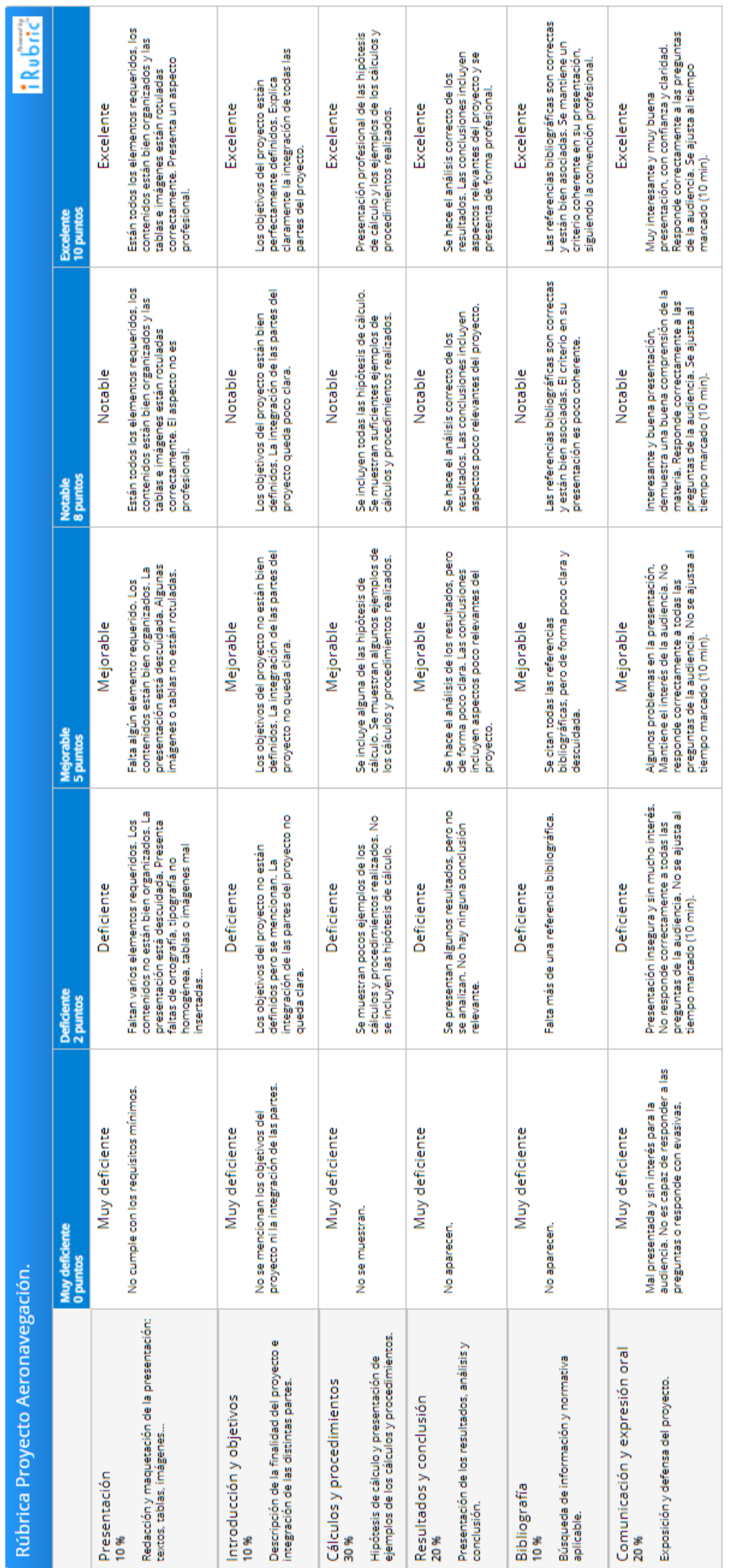

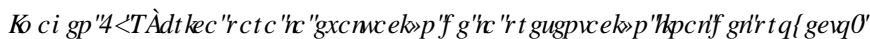

(cc) EY-NC-ND 2021, Universitat Politècnica de València

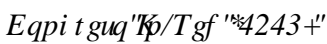




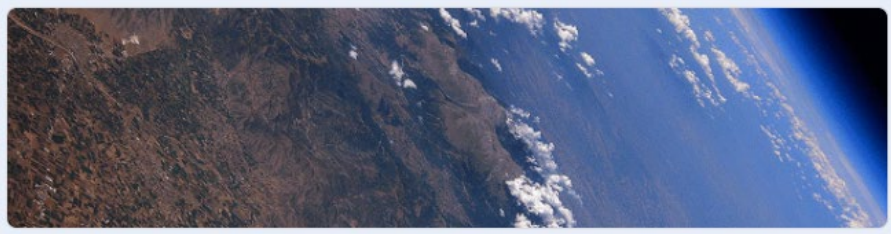

\section{ENCUESTA PROYECTO AERONAVEGACIÓN ETSID}

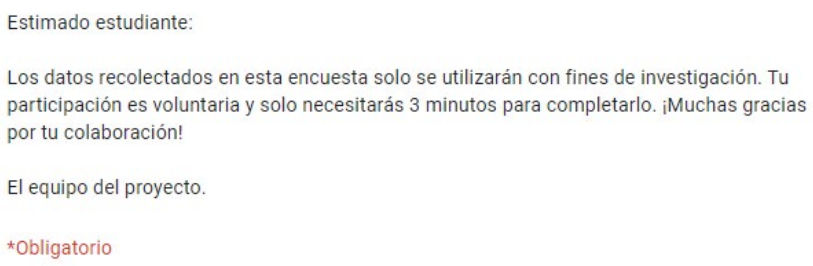

\begin{tabular}{ll}
\hline Cuestión & Valoración \\
\hline Grado de satisfacción con el proyecto realizado. & Poco (1) a Mucho (5) \\
\hline $\begin{array}{l}\text { Grado de satisfacción con el trabajo colaborativo realizado en varias de las } \\
\text { actividades del proyecto. }\end{array}$ & Poco (1) a Mucho (5) \\
\hline $\begin{array}{l}\text { Grado de satisfacción con la labor del profesorado. } \\
\text { La realización de las actividades del proyecto me ha resultado de gran ayuda en } \\
\text { el proceso de aprendizaje de las asignaturas. }\end{array}$ & Poco (1) a Mucho (5) \\
\hline
\end{tabular}

La realización del proyecto me ha ayudado a demostrar mis conocimientos. $\quad$ Desacuerdo (1) a Acuerdo (5)

La realización del proyecto me ha ayudado a mejorar mis habilidades (trabajo en grupo, toma de decisiones, resolución de problemas...) 


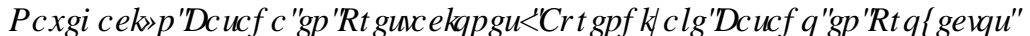 SDWHWKGDQWWIGHS HRQDYHDFIYQ]}

\section{Conclusiones}

Teniendo en cuenta que el fin último de este proyecto es facilitar el aprendizaje de las materias de la tecnología específica de Aeronavegación, mediante el uso de la herramienta metodológica de ABP, y motivar al alumnado en el proceso de aprendizaje de estas materias; se pretende mejorar este proceso en dos aspectos: por un lado, la integración de los conocimientos aportados por diferentes asignaturas, para conseguir que el estudiante las vea como parte de un conjunto y no de manera aislada, encontrando soluciones a un proyecto propuesto de forma que se asimilen los conceptos que forman parte de la teoría; y por otro lado, se pretende trabajar en el desarrollo de proyectos completos, lo cual será útil para trabajar la adquisición de las competencias necesarias en su futura vida profesional como ingenieros.

A pesar de que no podemos elaborar unas conclusiones garantes del logro de los objetivos de este proyecto, se ha podido observar que, sin duda, la metodología de ABP potencia la implicación del alumnado en su proceso de aprendizaje al trabajar con proyectos reales y de cierta complejidad.

Este proyecto es fácilmente transferible a otras tecnologías similares y, por supuesto, a las otras dos tecnologías específicas de la titulación de Grado en Ingeniería Aeroespacial. Se trata de asignaturas de especialización repartidas en los dos últimos cursos, por lo que se espera un grado de madurez e implicación del alumnado suficientemente alto como para garantizar el éxito de la experiencia.

\section{Agradecimientos}

Este trabajo está enmarcado en el Proyecto de Innovación y Mejora Educativa PIME/19-20/196 con título: "Navegación Basada en Prestaciones (Performance Based Navigation)", del Vicerrectorado de Estudios, Calidad y Acreditación de la Universitat Politècnica de València, siendo esta la entidad financiadora (UPV: Convocatoria Aprendizaje + Docencia. Proyectos de Innovación y Mejora Educativa)

\section{Referencias}

(UPV, 2021a) UNIVERSITAT POLITĖCNICA DE VALÈNCIA. * UDRIHQ, QJ HQIHAD\$ HRHSDFIDOI3 UHMQWFFYQ $<$ http://www.upv.es/titulaciones/GIA/indexc.html $>$ [Consulta: 18 de marzo de 2021]

(UPV. 2021b) UNIVERSITAT POLITĖCNICA DE VALÈNCIA. * WCRIHQ, QJ HQIHUDS HRHSDFIDOI\$ UJQDUKDVII $<$ http://www.upv.es/titulaciones/GIA/menu 1014617c.html> [Consulta: 18 de marzo de 2021]

(Fernández, 2006) FERNANDEZ MARCH, A. (2006). «Metodologías activas para la formación de competencias » en ( GXFDURUUJ

(García, 2017) GARCÍA MARTÍN, J. y PÉREZ-MARTÍNEZ, J.E. (2017). « Aprendizaje basado en proyectos: método para el diseño de actividades ». 7HFQRQR tDI\&IHQFW( GXFDFly Q\&( ) , vol 10, p. 37-63. https://www.tecnologia-cienciaeducacion.com/index.php/TCE/issue/view/21

(ICE-UPV, 2021) INSTITUTO DE CIENCIAS DE LA EDUCACIÓN - UNIVERSITAT POLITÈCNICA DE

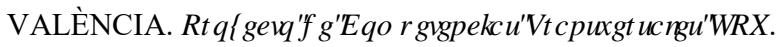

$<$ https://www.upv.es/entidades/ICE/info/Proyecto Institucional CT.pdf $>$ [Consulta: 21 de marzo de 2021]

(López, 2019) LÓPEZ-FERNÁNDEZ, D.; EZQUERRO, J.M.; RODRÍGUEZ, J.; PORTER, J. y LAPUERTA, V. (2019). « Motivational impact of active learning methods in aerospace engineering students » en $\square F W D \$ W W R Q D X W D$, vol. 165 , p. 344-354.

(Maldonado, 2008) Maldonado Pérez, M. (2008). « Aprendizaje Basado en Proyectos Colaborativos: Una experiencia en educación superior » en / DXUV, vol. 14, issue 28, p. 158-180. 


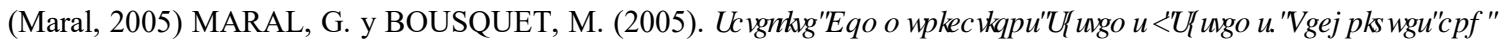
$7 H K Q R Q R J \mid 4$ th ed., John Wiley \& Sons.

(McGunagle, 2018) MCGUNAGLE, D. y ZIZKA, L. (2018). « Meeting Real World Demands of the Global Economy: An Employer's Perspective » en!-RXLQDORIIS YIDURQ\$ HRISDFH( GXFDMRQI [5 HHDUFKIvol. 27, issue 2, p. 59-76.

(OACI, 1983) ORGANIZACIÓN DE AVIACIÓN CIVIL INTERNACIONAL (1983). 0 DQXDOGH SURIHWRL $G H$

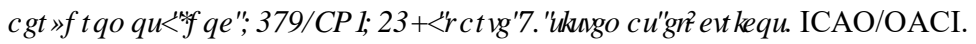

(OACI, 2004) ORGANIZACIÓN DE AVIACIÓN CIVIL INTERNACIONAL (2004). O DQXDO GH GUKR GH

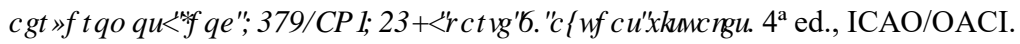

(OACI, 2006a) ORGANIZACIÓN DE AVIACIÓN CIVIL INTERNACIONAL (2006). O DQXDOGH GLMKR GH

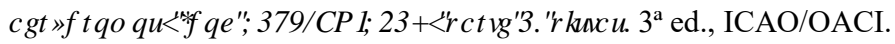

(OACI, 2006b) ORGANIZACIÓN DE AVIACIÓN CIVIL INTERNACIONAL (2006). 3 LRFHAPIHQWRVSDWD RVV

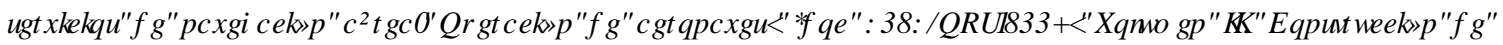
SLRFHIP IHQURVGHIXHRTILXDO ISRUTQWUXP HQWRV. $5^{\mathrm{a}}$ ed., ICAO/OACI.

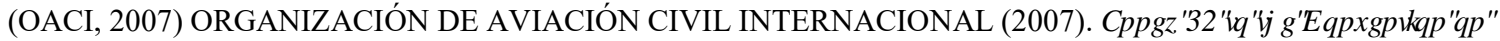

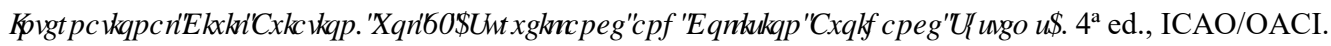

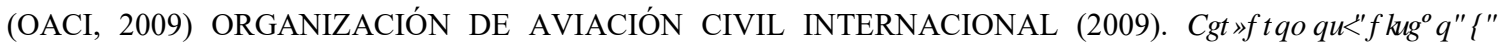
RSHDFIRQHZGHDHy GRP RVI9ROP HQ, . $5^{\mathrm{a}}$ ed., ICAO/OACI.

(OACI, 2011) ORGANIZACIÓN DE AVIACIÓN CIVIL INTERNACIONAL (2011). 0 DQXDO GH YIJIDQRED

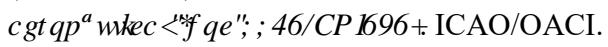

(Pisacane, 2008) PISACANE, V.L. y AMERICAN INSTITUTE OF AERONAUTICS (2008). 7KH6SDFH( QMIRQP HQW $D Q G$, Wh( IIHFWRQ6SDFH6। WWP V American Institute of Aeronautics and Astronautics.

(Prince, 2004) PRINCE, M. (2004). «Does Active Learning Work? A Review of the Research » en!-RXLQDORI ( QJLHUQJL( GXFDMRQIvol. 93, issue 3, p. 223-231.

(Rodríguez, 2015) RODRÍGUEZ, J.; LAVERÓN-SIMAVILLA, A.; EZQUERRO, J.M.; DEL CURA, J.M.; LAPUERTA, V. y CORDERO-GARCÍA, M. (2015). «Project Based Learning experiences in the space engineering education at Technical University of Madrid » en \$ GDQFHMIQ6SDFH5 HMDUF, vol. 56, p. 1319-1330.

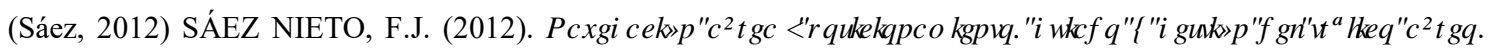
Ibergarceta.

(Tippelt, 2001) TIPPELT, R. y LINDEMANN, H. (2001). «El método de Proyectos ».

http://132.248.239.10/cursos diplomados/diplomados/basico/colima07/5 material didactico/productos didac/metproy.pdf

(Zabalza, 2011) ZABALZA BERAZA, M.A. (2011). «Metodología docente » en[5 HLWW GH' RFHQFD8 QIYHWWUDD

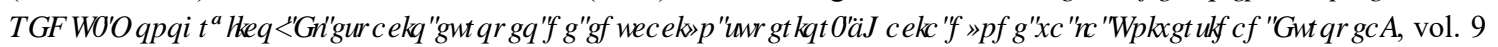
(3), p. 75-98. 